\title{
THE SPECTRUM OF A PARTIALLY IONIZED JET
}

\author{
L. Binette ${ }^{1}$, A. Raga $^{1}$, J. Canto $^{2}$ \\ ${ }^{1}$ Institute for Theoretical Astrophysics, Canada \\ ${ }^{2}$ Universidad Nacional Autónoma de México, México
}

Observed stellar jets have the following characteristics : a high degree of collimation, a structure of bright, quasi-periodic knots, a very low excitation spectrum (red [S II] to $\mathrm{H} \alpha$ line ratios of 1-10). Assuming that the latter are indicative of the structure of a supersonic jet, we have made a detailed prediction of the emission line spectrum within each radiating knot using the multi-purpose code MAPPINGS which includes all the atomic processes important in low velocity shocks. The structure (density, temperature, ionization) of the stationary, supersonic, nonadiabatic stellar jet was computed separately using a hydrodynamical code developed by $A$. Raga. An initially overpressured supersonic jet tries to adjust its pressure to the environmental pressure. In this process of pressure adjustment, a series of expansion fans and incident/reflected shock pairs are formed along the jet. We associate the emission from the recombination region behind these crossing shocks with the knots observed in stellar jets. We find that the calculated emission spectrum is of remarkably low excitation.

\section{SODIUM IONIZATION IN T-TAURI STARS}

\author{
A. Natta ${ }^{1}$ and C. Giovanardi ${ }^{2}$ \\ Osservatorio Astrofisico di Arcetri, Italy \\ Centro per l'Astronomia Infrarossa, CNR, Italy
}

We present the results of a study of the sodium ionization and excitation in the winds of low-luminosity, pre-main-sequence stars. Line profiles for the NaI doublet at 5990,5986 Åare discussed and compared with the observations for those $T$ Tauri stars with P-Cygni profiles. We find that the observed shape of the lines put significative constraints on the rate of mass-loss $\left(M \geq 3 \times 10^{-8} M_{\odot} / y r\right)$.

By comparing the properties of the $\mathrm{NaI}$ lines with the observed luminosity of infrared hydrogen recombination lines, such as $\mathrm{Br}_{7}$, it is possible to determine at the same time the rate of mass-loss and the temperature of the inner regions of the wind, where both lines are formed. The results for four stars show that the wind is in general cool $\left(T_{g a s} \$ 7000 \mathrm{~K}\right)$, and that the momentum in the wind is comparable to the momentum measured from $\mathrm{CO}$ lines. 